\title{
Análise da Concentração de Material Particulado (Mp2,5) na Instalação e 0peração do Corredor Viário Transolímpica
}

\author{
Diego Rafael dos Santos Peixotol'; Simone Lorena Quitério de Souza²; \\ Paulo Sergio de Oliveira Cezario

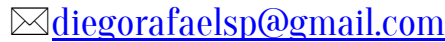 \\ 1. Universidade do Estado do Rio de Janeiro - RJ. \\ 2. Instituto Federal de Educação, Ciência e Tecnologia do Rio de Janeiro - RJ
}

Histórico do Artigo:

Recebido em: 24 de marco de $2020 \quad$ Aceito em: 27 de abril de $2020 \quad$ Publicado em: 30 de abril de 2020

Resumo: 0 material particulado fino $\left(\mathrm{MP}_{2,5}\right)$ é um dos principais poluentes que contribuem para a diminuição da qualidade do ar em áreas sob influência de rodovias. Devido ao seu tamanho, o $\mathrm{MP}_{2,5}$ pode penetrar nas camadas mais profundas do sistema respiratório causando problemas de saúde na população exposta ao poluente. Na Transolímpica, um corredor viário do Rio de Janeiro, o monitoramento de $\mathrm{MP}_{2,5}$ foi realizado durante a fase de instalação e operação da via por exigência do órgão ambiental fiscalizador, o Instituto Estadual do Ambiente (INEA). 0 presente trabalho analisou a concentração de $\mathrm{MP}_{2.5}$, em duas estações de monitoramento da qualidade do ar próximas à Transolímpica, no período de outubro de 2014 a outubro de 2018, contemplando as fases de instalação e operação da via. Foi realizada a comparação da concentração de $\mathrm{MP}_{2,5}$ entre as fases de instalação e operação, com os limites estabelecidos pela Resolução CONAMA No 491/2018 e pela Organização Mundial da Saúde (OMS). Além disso, as variáveis meteorológicas foram correlacionadas com a concentração de $\mathrm{MP}_{2.5}$ obtida no período. $0 \mathrm{~s}$ resultados mostraram que o limite da Resolução CONAMA N 491/2018 foi extrapolado três dias na fase de instalação e o limite recomendado pela OMS foi violado em 81 dias de amostragem. A comparação entre as fases de instalação e operação da Transolímpica mostrou que durante as obras a concentração de $\mathrm{MP}_{2.5}$ foi cerca de $24 \%$ maior. E todas as variáveis meteorológicas se mostraram fracamente correlacionadas com a concentração de $\mathrm{MP}_{2,5}$. Palavras-chave: Material particulado fino, Qualidade do ar, Rodovias, $\mathrm{MP}_{2,5}$.

\section{Particulate Matter Concentration $\left(\mathrm{Pm}_{2.5}\right)$ Analysis in the Installation and Operation of the Transolímpica Road Corridor}

\begin{abstract}
Fine particulate material $\left(\mathrm{PM}_{2.5}\right)$ is one of the main pollutants that contributes to the air quality decrease in the highways influence areas. Due to its size, $\mathrm{PM}_{2.5}$ can penetrate deeper in the respiratory system layers, causing health problems in the population exposed to the pollutant. At Transolímpica, a highway in Rio de Janeiro, $\mathrm{PM}_{2.5}$ monitoring was carried out during the road installation and operation, as required by the environmental agency, the Instituto Estadual do Ambiente (INEA). The present study analyzed $\mathrm{PM}_{2.5}$ concentration in two air quality monitoring stations neart by Transolímpica, from October 2014 to October 2018, contemplating installation and operation road phases. $\mathrm{PM}_{2.5}$ concentration was compared between installation and operation phases, with Resolução CONAMA N ${ }^{\circ}$ 491/2018 and the World Health Organization (WHO) standards. In addition, meteorological variables were correlated with $\mathrm{PM}_{2.5}$ concentration obtained in the period. The results showed that the Resolução CONAMA $\mathrm{N}^{\circ} 491 / 2018$ limit of was extrapolated three days in installation phase and the recommended WHO standard was violated in 81 days of sampling. The comparison between the Transolímpica installation and operation phases showed that during the construction $\mathrm{PM}_{2.5}$ concentration was about $24 \%$ higher. And all variables were poorly correlated with $\mathrm{PM}_{2.5}$ concentration.
\end{abstract}

Keywords: Fine particulate matter, Air quality, Roads, $\mathrm{PM}_{2.5}$. 


\section{Análisis de la Concentración de Material Particulado (Mp2.5) en la Instalación y Operación del Corredor de Carretera Transolímpico}

Resumen: El material particulado fino $\left(\mathrm{MP}_{2.5}\right)$ es uno de los principales contaminantes que contribuyen a la disminución de la calidad del aire en áreas bajo la influencia de las carreteras. Debido a su tamaño, $\mathrm{MP}_{2.5}$ puede penetrar en las capas más profundas del sistema respiratorio causando problemas de salud en la población expuesta al contaminante. En Transolímpica, un corredor vial en Rio de Janeiro, el monitoreo de $\mathrm{MP}_{2.5}$ se llevó a cabo durante la fase de instalación y operación de la carretera, según lo requerido por el organismo de inspección ambiental, el Instituto Estadual do Ambiente (INEA). El presente trabajo analizó la concentración de $\mathrm{MP}_{2.5}$ en dos estaciones de monitoreo de la calidad del aire cerca de la Transolímpica, de octubre de 2014 a octubre de 2018, cubriendo las fases de instalación y operación de la carretera. Se realizó una comparación de la concentración de $\mathrm{MP}_{2.5}$ entre las fases de instalación y operación, con los límites establecidos por la Resolución CONAMA N ${ }^{\circ}$ 491/2018 y por la Organización Mundial de la Salud (0MS). Además, las variables meteorológicas se correlacionaron con la concentración de $\mathrm{MP}_{2.5}$ obtenida en el período. Los resultados mostraron que el límite de la Resolución CONAMA N ${ }^{\circ}$ 491/2018 fue extrapolado tres días en la fase de instalación y el límite recomendado por la 0MS fue violado en 81 días de muestreo. La comparación entre las fases de instalación y operación de Transolímpica mostró que durante los trabajos la concentración de $\mathrm{MP}_{2.5}$ fue aproximadamente un $24 \%$ mayor. Y todas las variables meteorológicas se correlacionaron débilmente con la concentración de $\mathrm{MP}_{2.5}$.

Palabras clave: Partículas finas, Calidad del aire, Carreteras, $\mathrm{MP}_{2.5}$.

\section{INTRODUÇ̃̃o}

A alteração da qualidade do ar é um dos principais impactos ambientais decorrentes das fases de instalação e operação de rodovias (SÁNCHEZ, 2013). Dentre os diversos poluentes emitidos para atmosfera nessas fases, o material particulado fino aparece como um dos mais relevantes (MING et al., 2017). 0 material particulado fino consiste em uma complexa mistura de partículas sólidas e líquidas na forma de aerossóis, poeira, metais, fuligem, entre outros, que possui diâmetro aerodinâmico de até 2,5 $\mu \mathrm{m}$ (BRASIL, 2018).

$0 \mathrm{MP}_{2,5}$ tem sido alvo de grande preocupação devido a sua capacidade de penetrar nas vias respiratórias inferiores e causar, principalmente, doenças respiratórias e cardíacas (GUARIEIR0 et al., 2011). Segundos os estudos desenvolvidos por Jerret (2015) e Lelieveld et al. (2015), o material particulado fino foi responsável por mais de 3 milhões de mortes prematuras no ano de 2015.

Entretanto, apenas no ano de 2018 foi regulamentada no Brasil a primeira legislação estabelecendo padrões limites de emissão de $\mathrm{MP}_{2,5}$ para a atmosfera em nível federal. A Resolução CONAMA Nº 491/2018 está sendo implementada em etapas, por isso, o padrão intermediário que vigora, provisoriamente, é de $60 \mu \mathrm{g} \mathrm{m} \mathrm{m}^{-3}$ para o período de 24 horas e de $20 \mu \mathrm{g}$ $\mathrm{m}^{-3}$ considerando a média de concentração anual. Como padrão final está previsto a adoção dos 
Análise da Concentração de Material Particulado $(\mathrm{Mp} 2,5)$ na Instalação e Operação do Corredor Viário Transolímpica

valores recomendados pela Organização Mundial da Saúde (0MS), de $25 \mu \mathrm{g} \mathrm{m} \mathrm{m}^{-3} \mathrm{~d}^{-1} \mathrm{e} 10 \mu \mathrm{g} \mathrm{m} \mathrm{m}^{-3} \mathrm{a}^{-}$ ${ }^{1}$ (BRASIL, 2018).

A Transolímpica é um corredor viário de $26 \mathrm{~km}$ de extensão que liga os bairros de Deodoro a Barra da Tijuca. Essa via expressa, que começou a ser construída em 2012 e a operar no ano de 2016, teve seu processo de licenciamento ambiental conduzido pelo Instituto Estadual do Ambiente (INEA), órgão ambiental licenciador no estado do Rio de Janeiro.

Durante o licenciamento foi exigido pelo INEA ações de monitoramento da qualidade do ar com a operação de amostradores de grande volume de partículas em suspensão (HI-VOL). Para $\mathrm{MP}_{2,5}$ foram instalados HI-VOL em dois locais, Estações de Monitoramento da Qualidade do $\operatorname{Ar}(\mathrm{EMQA})$ Bosque da Boiuna e Rio Grande que operaram desde a fase de obras até a operação da Transolímpica (INEA, 2013).

Este trabalho tem como objetivo analisar e comparar as concentrações de $\mathrm{MP}_{2,5}$ obtidas durante a fase de instalação e operação da Transolímpica nas EMQ̧A Bosque da Boiuna e Rio Grande. E, além disso, correlacionar os dados de concentração de $\mathrm{MP}_{2,5}$ com variáveis meteorológicas visando avaliar a influência dessas variáveis na concentração de material particulado.

\section{MATERIAL E MÉTODOS}

0 local de estudo se encontra na Bacia Aérea II que compreende os bairros de Jacarepaguá e Barra da Tijuca, localizados no município do Rio de Janeiro - RJ, e possui uma área de, aproximadamente, $182 \mathrm{~km}^{2}$ (MARTINS et al., 2014).

Os dados de concentração de $\mathrm{MP}_{2,5}$ foram obtidos de duas estações semiautomáticas de monitoramento operadas pela empresa responsável pela gestão ambiental da Transolímpica, de acordo com a Resolução CONAMA Nº 03/1990 vigente a época, durante o período de 14 de outubro de 2014 a 28 de outubro de 2018. A Estação de Monitoramento da Qualidade do Ar (EMQQA) Bosque da Boiuna está localizada, no bairro da Taquara, no interior do condomínio de mesmo nome, a cerca de 400 metros da Transolímpica (2254'50.35”S 4323’45.08”0). Enquanto que a EMQA Rio Grande está localizada a cerca de 100 metros da Transolímpica, na estrada do Rio Grande, no bairro de Jacarepaguá, em frente ao condomínio Golden Dreams (2255’10.67”S 43²3’55.61”0). A localização das EMQA pode ser observada na Figura 1. 


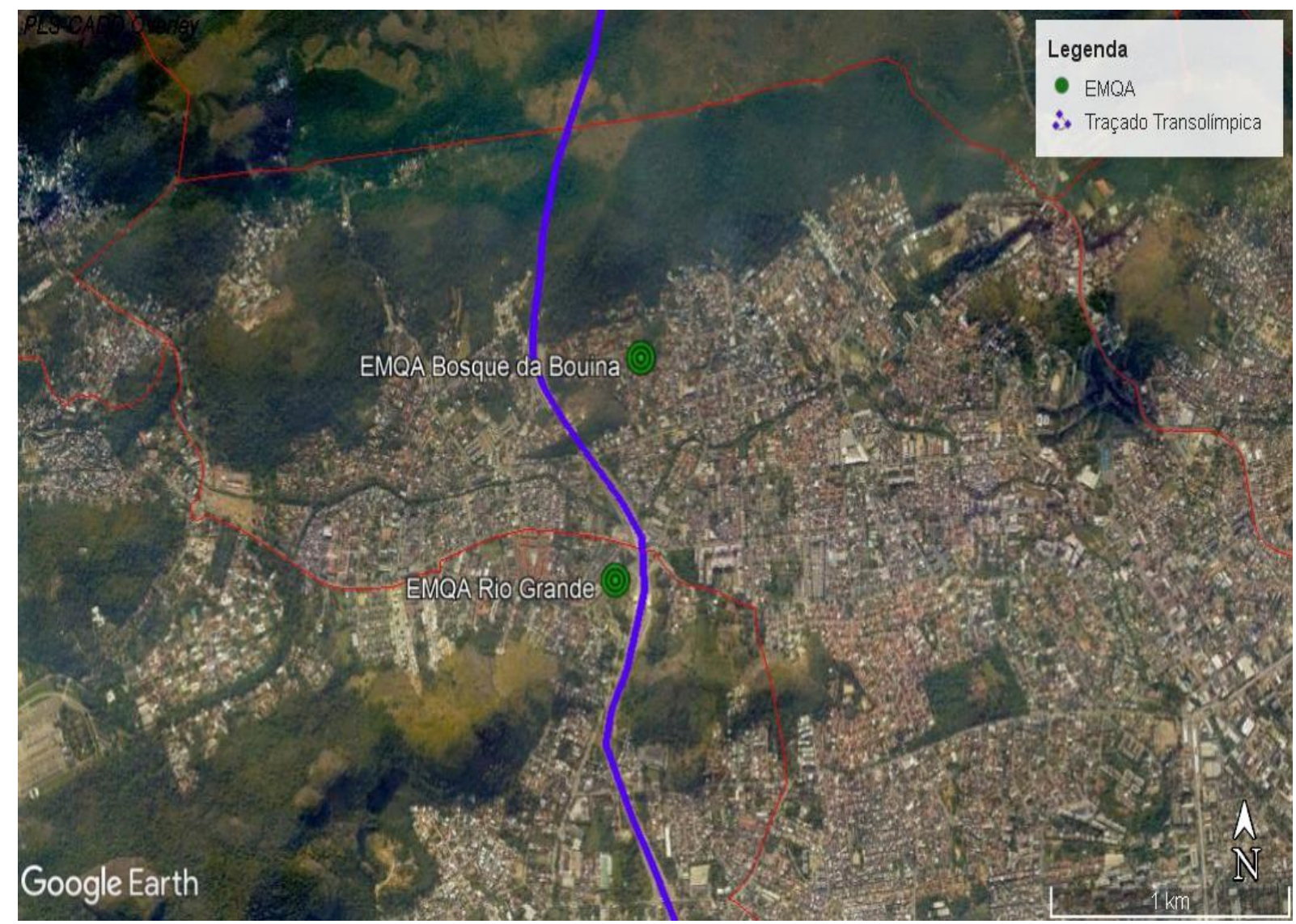

Figura 1 - Localização das Estações de Monitoramento da Qualidade do Ar (EMQA) Bosque da Boiuna e Rio Grande em relação ao traçado da Transolímpica.

Fonte: Google Earth Pro (2018).

A seleção dos dados foi realizada considerando a frequência de amostragem de seis em seis dias durante todo o período analisado. Entretanto, algumas medições, referentes aos dias em que os equipamentos amostradores apresentaram falhas técnicas, foram desconsideradas. Dessa forma, para este estudo foram considerados os dados de 106 dias amostrados durante a fase de instalação e 140 dias amostrados durante a fase de operação, em ambas as EMQA.

Todo o monitoramento foi realizado utilizando o amostrador de grande volume (AGV $\mathrm{MP}_{2,5}$ ), fabricado pela empresa Energética Qualidade do Ar, e a metodologia seguiu o recomendado pela ABNT NBR 13412/1995 (DIAS, 2017).

Os dados meteorológicos diários, foram obtidos, durante todo o período em que ocorreram as atividades de monitoramento, da Estação Meteorológica do Aeroporto de Jacarepaguá - Roberto Marinho, considerando os seguintes parâmetros: temperatura média $\left({ }^{\circ} \mathrm{C}\right)$, umidade relativa média $(\%)$, precipitação total $(\mathrm{mm})$, pressão média no nível da estação (hPa) e velocidade do vento máximo (Kt).

Inicialmente os valores de concentração diária de $\mathrm{MP}_{2,5}$ foram comparados com os padrões estabelecidos pela Resolução CONAMA N 491/2018 e recomendados pela Organização 
Análise da Concentração de Material Particulado $(\mathrm{Mp} 2,5)$ na Instalação e 0peração do Corredor Viário Transolímpica

Mundial da Saúde (0MS). Para verificar se houve diferença na concentração de $\mathrm{MP}_{2,5}$ entre as fases de instalação e operação da Transolímpica foi aplicado o teste Mann-Whitney nos dados de cada EMQA. E a correlação de Spearman foi usada para verificar a correlação entre o $\mathrm{MP}_{2,5} \mathrm{e}$ as variáveis meteorológicas. Todas as análises estatísticas, descritiva e multivariada, foram realizadas por meio do software $R$ Studio.

\section{RESULTADOS E DISCUSSÃ0}

Comparando as concentrações de $\mathrm{MP}_{2,5}$ com o padrão intermediário estipulado pela Resolução CONAMA No 491/2018 e com o padrão recomendado pela OMS para o período de 24 horas foi possível observar que na fase de instalação o limite da Resolução CONAMA No 491/2018 $\left(60 \mu \mathrm{g} \mathrm{m} \mathrm{m}^{-3}\right.$ ) foi violado apenas na EMQA Rio Grande (Gráfico 1(a)), em três dos 106 dias amostrados. Entretanto, o valor de $25 \mu \mathrm{g} \mathrm{m}^{-3}$, recomendado pela 0MS, foi ultrapassado em 25 dias na EMQA Rio Grande e em 24 dias na EMQA Bosque da Boiuna (Gráfico 2(a)).

Na fase de operação, ambas as EMQQA apresentaram concentrações de $\mathrm{MP}_{2,5}$ abaixo de 60 $\mu \mathrm{g} \mathrm{m}^{-3}$ (Resolução CONAMA No 491/2018), porém em 14 dos 140 dias em que ocorreu amostragem foi ultrapassado o limite recomendado pela OMS na EMQA Rio Grande (Gráfico 1(b)) e na EMQA Bosque da Boiuna a concentração de $25 \mu \mathrm{g} \mathrm{m}^{-3}$ foi ultrapassada em 18 dias (Gráfico 2(b)).

As concentrações diárias de $\mathrm{MP}_{2,5}$ obtidas durante a fase de operação são semelhantes as encontradas durante 0 monitoramento realizado em 2016 nos bairros Recreio dos Bandeirantes (2 a $\left.36 \mu g \mathrm{~m}^{-3}\right)$, Copacabana (3 a $\left.29 \mu \mathrm{g} \mathrm{m} \mathrm{m}^{-3}\right)$, Urca (3 a $23 \mu \mathrm{g} \mathrm{m} \mathrm{m}^{-3}$ ) e Lagoa (1 a $28 \mu \mathrm{g}$ $\mathrm{m}^{-3}$ ) conforme mostra o estudo realizado por Ventura et al. (2019). 
Gráfico 1 - Comparação da concentração de $\mathrm{MP}_{2,5}$ na EMQA Rio Grande

\section{(a) EMQA Rio Grande - Fase de Instalação}

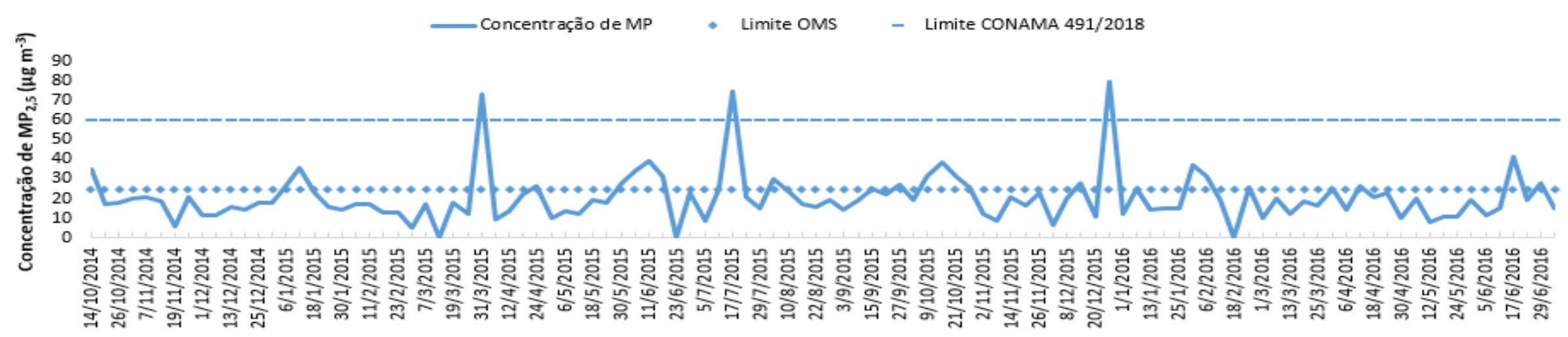

Data da amostragem

\section{(b) EMQA Rio Grande - Fase de Operação}

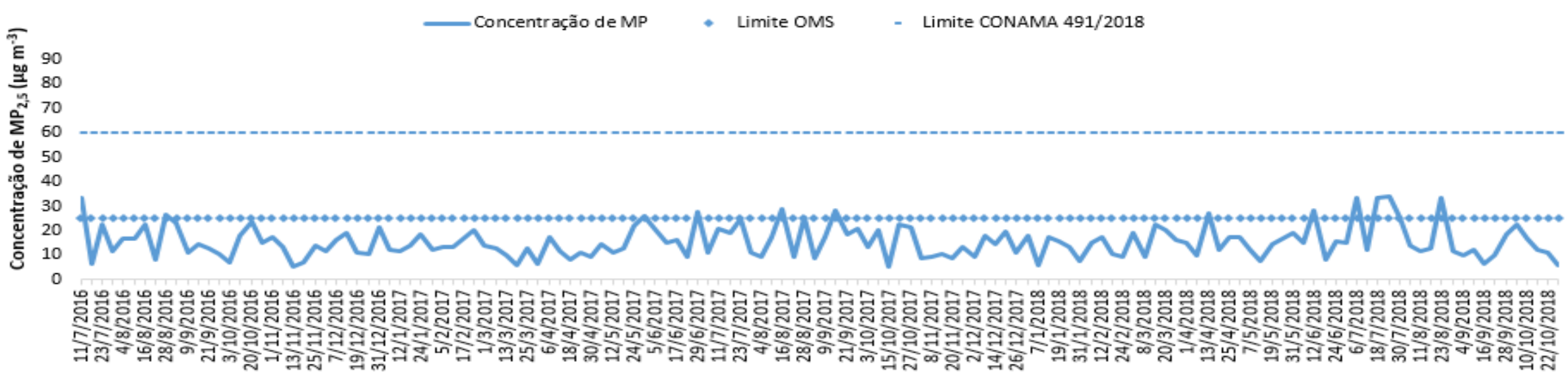

Data da amostragem

Fonte: 0s Autores. 
Gráfico 2 - Comparação da concentração de $\mathrm{MP}_{2,5}$ na EMQQA Bosque da Boiuna

\section{(a) EMQA Bosque da Boiuna - Fase de Instalação}

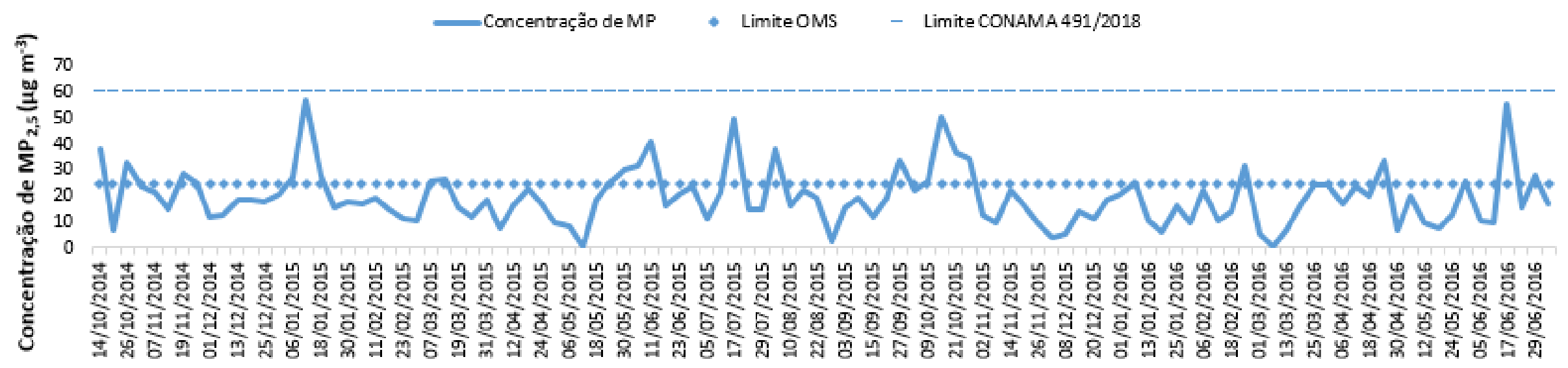

Data da amostragem

\section{(b) EMQA Bosque da Boiuna - Fase de Operação}

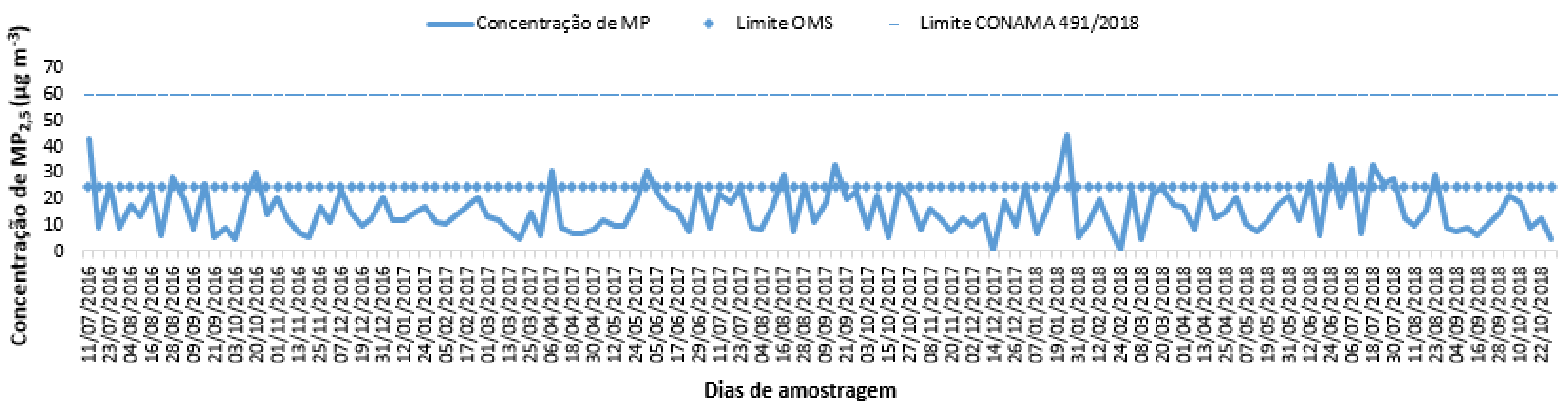

Fonte: 0s Autores.

Revista Internacional de Ciências, v. 10, n. 01, p. 124-138, jan-abr, 2020 
A Tabela 1 mostra o resumo da estatística descritiva dos dados da concentração de $\mathrm{MP}_{2,5}$, nas fases de instalação e operação, tanto na EMQQA Rio Grande quanto na EMQ̧A Bosque da Boiuna. Observa-se que a maior concentração de $\mathrm{MP}_{2,5}$ foi obtida na EMQA Rio Grande, durante a fase de instalação, e a menor na EMQ్ Bosque da Boiuna, também na fase de instalação da Transolímpica. A média acompanhou o mesmo padrão, apresentando maiores valores na fase instalação, sendo o maior na EMQ_A Rio Grande e menores valores nas fases de operação.

Essa diferença observada entre as EMQA, especialmente na fase de instalação, era esperada, uma vez que a EMQA Rio Grande está localizada mais próxima ao local de obras para a construção da Transolímpica quando comparada a EMQA Bosque da Boiuna que está inserida dentro de um condomínio, mais afastada de pontos de intervenção da obra (Tabela 1).

Tabela 1 - Estatística descritiva dos dados

\begin{tabular}{|c|c|c|c|c|}
\hline $\begin{array}{c}\text { Estatística } \\
\text { Descritiva }\end{array}$ & $\begin{array}{c}\text { EMQA Bosque da } \\
\text { Boiuna } \\
\text { Instalação }\end{array}$ & $\begin{array}{c}\text { EMQA Bosque da } \\
\text { Boiuna 0peração }\end{array}$ & $\begin{array}{c}\text { EMQA Rio } \\
\text { Grande } \\
\text { Instalação }\end{array}$ & $\begin{array}{c}\text { EMQA Rio } \\
\text { Grande 0peração }\end{array}$ \\
\hline $\begin{array}{c}\text { Concentração } \\
\text { mínima ( } \mu \mathrm{g} \mathrm{m}^{-3} \text { ) }\end{array}$ & 2,58 & 4,34 & 5,44 & 5,22 \\
\hline $\begin{array}{c}\text { Concentração } \\
\text { média }\left(\mu \mathrm{g} \mathrm{m}^{-3}\right)\end{array}$ & 19,3 & 15,6 & 20,4 & 15,5 \\
\hline $\begin{array}{c}\text { Concentração } \\
\text { máxima }\left(\mu \mathrm{g} \mathrm{m}^{-3} \text { ) }\right.\end{array}$ & 56,82 & 44,93 & 79,98 & 33,76 \\
\hline
\end{tabular}

Fonte: 0s Autores.

Ao comparar a concentração de $\mathrm{MP}_{2,5}$ da fase de instalação (Gráfico 3) com a da fase de operação (Gráfico 4) verifica-se que a concentração na instalação é cerca de $24 \%$ maior do que na operação, além disso, os picos de maiores valores de $\mathrm{MP}_{2,5}$ também foram mais altos na fase de instalação. Essa diferença se mostrou estatisticamente significativa $(p<0,05)$ para a EMQA Rio Grande $\left(w=9479\right.$ e $p-$ valor $\left.=1,369 e^{-05}\right)$ e para a EMQA Bosque da Boiuna $(w=8777$ e $p$-valor $=0,003816$ ), após a aplicação do teste de Mann-Whitney, que permite a comparação de dois conjuntos de dados que não apresentam distribuição normal.

Apesar da fase de obras gerar notadamente mais material particulado grosso, com diâmetro aerodinâmico de até $10 \mu \mathrm{m}\left(\mathrm{MP}_{10}\right)$, devido à movimentação de terra, ao carreamento de lama para vias públicas e à ressuspensão de poeira, a diferença observada na concentração de $\mathrm{MP}_{2,5}$ indica que as atividades de construção civil também alteram a concentração local de 
Análise da Concentração de Material Particulado $(\mathrm{Mp} 2,5)$ na Instalação e Operação do Corredor Viário Transolímpica

material particulado fino em suspensão (CHOWDHURI; GUPTA, 2017; KINSEY et al., 2004). A principal hipótese para explicar isso é que durante a fase de instalação o fluxo de veículos, principalmente pesados, como caminhões e máquinas, é muito maior em comparação com a fase de operação e tais veículos tendem a emitir partículas de $\mathrm{MP}_{2,5}$ por seus escapamentos (JUDAREZLER; REIZER; OUDINET, 2011; LIU et al., 2017). Segundo Godoy et al. (2018), no Rio de Janeiro, as emissões veiculares contribuem com $60 \%$ da concentração de $\mathrm{MP}_{2,5}$.

Gráfico 3 - Concentração de $\mathrm{MP}_{2,5}$ durante as fases de instalação e de operação na EMQQA Bosque da Boiuna

\section{EMQA Bosque da Boiuna}

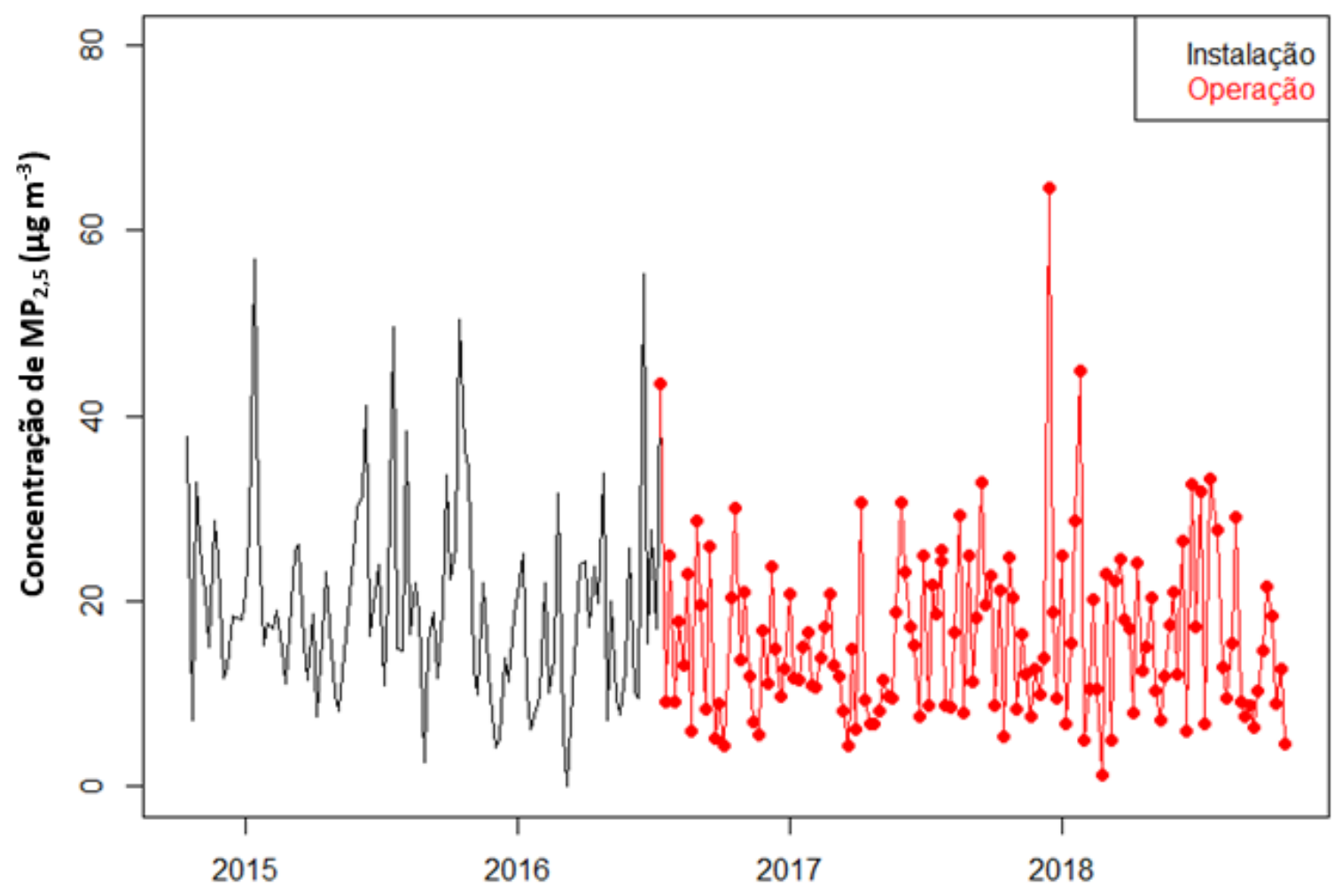

Fonte: 0s Autores. 
Gráfico 4 - Concentração de $\mathrm{MP}_{2,5}$ durante as fases de instalação e de operação na EMQูA Rio Grande.

\section{EMQA Rio Grande}

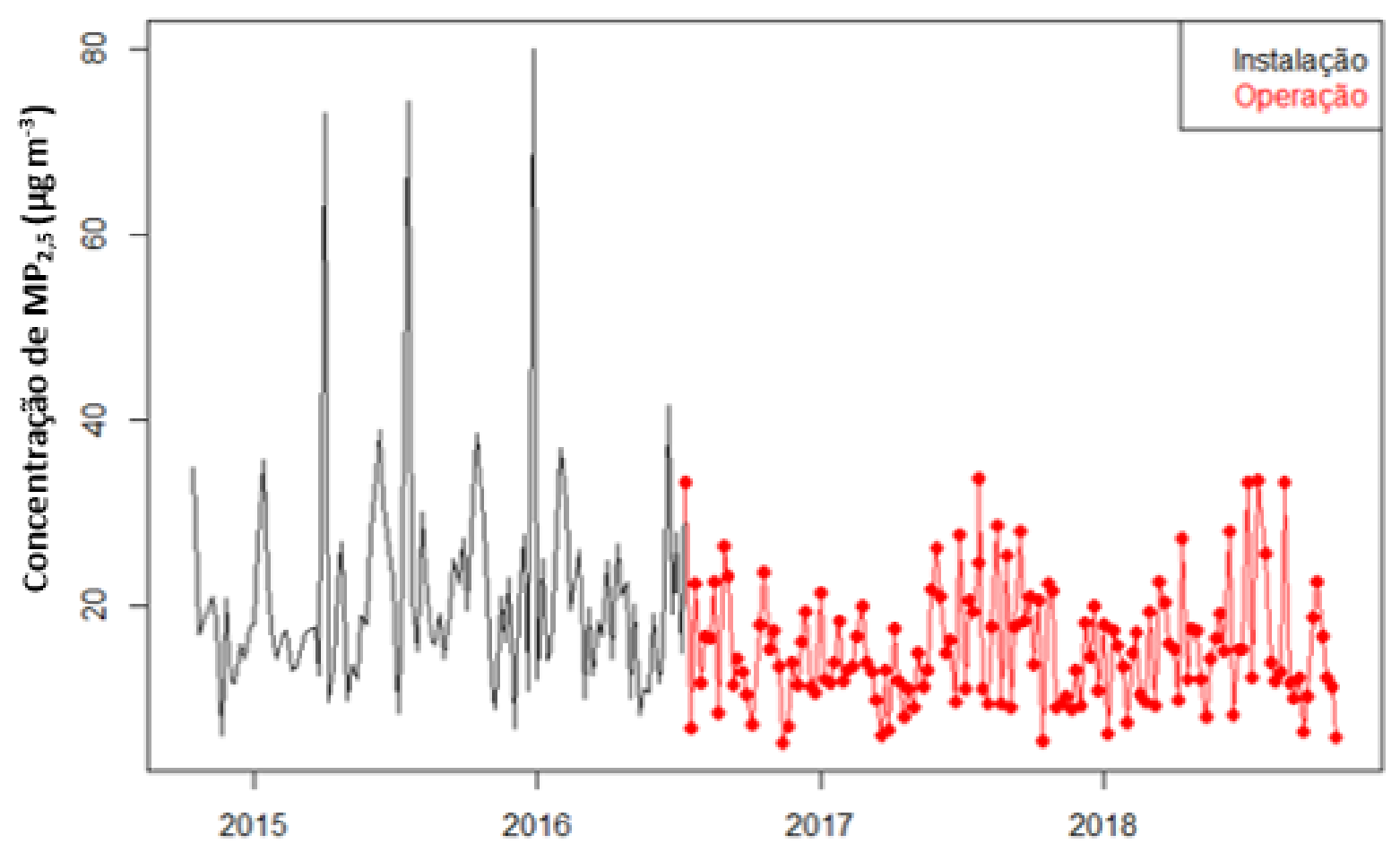

Fonte: 0s Autores

0 Gráfico 5 mostra a concentração média anual de $\mathrm{MP}_{2,5}$ durante as fases de instalação (IN) e operação (OP) da Transolímpica nas EMQQA Rio Grande e Bosque da Boiuna. Considerando os dois anos completos durante o período analisado, 2015 para a fase de instalação e 2017 para a fase de operação, verifica-se que o limite recomendado pela 0MS, de $10 \mu \mathrm{g} \mathrm{m} \mathrm{m}^{-3}$, foi ultrapassado em ambas as fases nas duas EMQA. Enquanto o limite anual estabelecido pela Resolução CONAMA $\mathrm{N}^{0}$ 491/2018 (20 $\left.\mu \mathrm{g} \mathrm{m}^{-3}\right)$, foi violado em ambas as EMQQA apenas na fase de instalação (Gráfico 5).

Nota-se a redução substancial da concentração anual de $\mathrm{MP}_{2,5}$ na fase de operação da Transolímpica, o que corrobora o entendimento de que as atividades de construção civil também contribuem para aumentar a quantidade de partículas de material particulado fino em suspensão na atmosfera.

Apesar dessa diminuição na concentração anual de $\mathrm{MP}_{2,5}$, ressalta-se que segundo a 0MS, não há valor limite que ao ser atingido forneça total proteção à saúde humana. Dessa forma, além de continuar o monitoramento, é importante que sejam adotadas políticas públicas de 
Análise da Concentração de Material Particulado $(\mathrm{Mp} 2,5)$ na Instalação e 0peração do Corredor Viário Transolímpica

redução das emissões de poluentes atmosféricos, como o incentivo ao uso de veículos com tecnologias menos poluentes.

Gráfico 5 - Concentração média anual de $\mathrm{MP}_{2,5}$

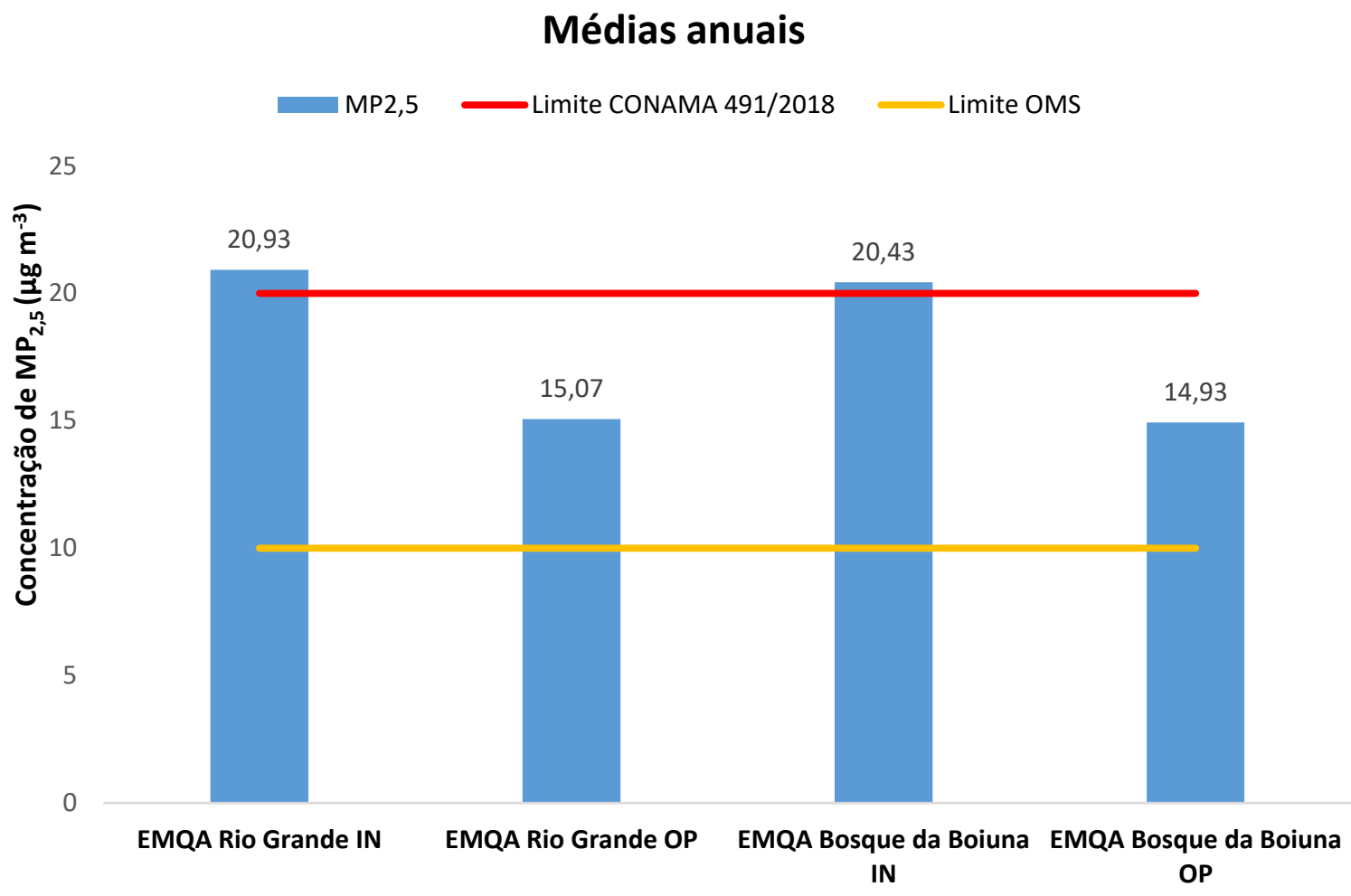

Fonte: Os Autores.

A Figura 2 mostra a correlação entre a concentração de $\mathrm{MP}_{2,5}$ (MP25) e as variáveis meteorológicas nas EMQA Rio Grande e Bosque da Boiuna durante as fases de instalação e de operação da Transolímpica, onde valores negativos representam uma correlação negativa, enquanto valores positivos representam uma correlação positiva. E quanto mais próximo de 1 ou -1 maior é a correlação.

A análise da influência de variáveis meteorológicas na concentração de $\mathrm{MP}_{2,5}$ mostrou, a partir dos valores do coeficiente de correlação de Spearman, que todas as variáveis consideradas apresentaram um padrão de correlação fraca com $0 \mathrm{MP}_{2,5}$ (MP25) tanto na fase de instalação quanto na fase de operação nas duas EMQA (Figura 2).

A precipitação atmosférica (Chuva) foi a variável que apresentou maior coeficiente de correlação com a concentração de MP2,5 (MP25). A correlação entre MP25 e Chuva apresentou um padrão inverso, ou seja, a concentração de $\mathrm{MP}_{2,5}$ tende a diminuir com o aumento dos valores de precipitação em ambas as fases e EMQA. 0 maior valor de coeficiente de correlação de 
Spearman encontrado foi na EMQA Rio Grande na fase da instalação, indicando que nessa fase a concentração de $\mathrm{MP}_{2,5}$ apresentou maior associação com a precipitação pluviométrica. A temperatura (Temp) apresentou um padrão de correlação positiva com MP25 nas fases de instalação e operação tanto na EMQQA Bosque da Boiuna quanto na EMQুA Rio Grande. Já umidade relativa (Ur) exibiu um padrão de correlação negativa com MP25, em ambas as EMQA nas fases de instalação e operação, o mesmo aconteceu com a pressão atmosférica (Press). Apenas a velocidade do vento (Vel) não apresentou um padrão comum a todas EMQQA nas fases de instalação e operação da via.

Mesmo com coeficientes fracos de correlação duas das variáveis meteorológicas analisadas (Ur e Chuva) apresentaram padrões condizentes com o que a literatura relata (GUERRA et al., 2011). Em geral, o aumento da umidade do ar contribui para a formação de nuvens e com isso para a ocorrência de precipitação pluviométrica. Além disso, o aumento da velocidade do vento e da temperatura elevam movimentos verticais ascendentes de convecção. Esses fenômenos tendem a dispersar e diluir os poluentes e, consequentemente, diminuir suas concentrações na atmosfera (VENTURA, 2015; MONTE et al., 2016).

Uma hipótese para explicar a fraca correlação entre a concentração de MP2,5 e as variáveis meteorológicas pode ser a distância entre a estação meteorológica do Aeroporto de Jacarepaguá, cujos dados foram obtidos, e as EMQQA. Essa distância, de cerca de 8 km, diminui a probabilidade de registro das variações climáticas locais nos pontos onde ocorreu 0 monitoramento. 

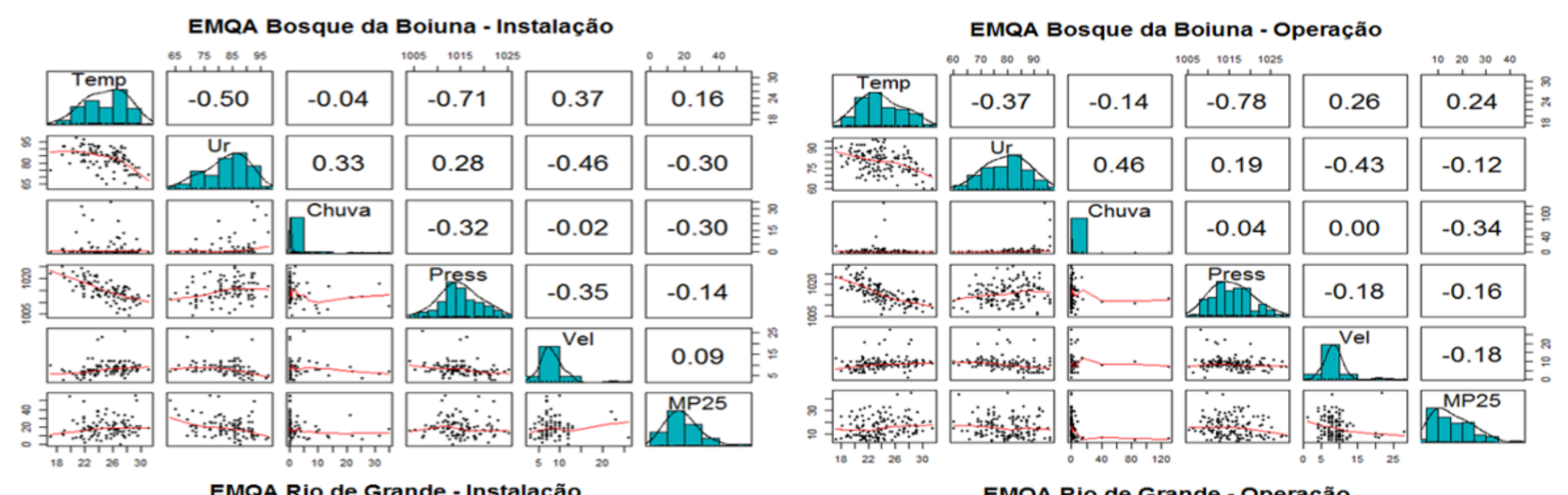

\section{EMQA Rio de Grande - Instalação}
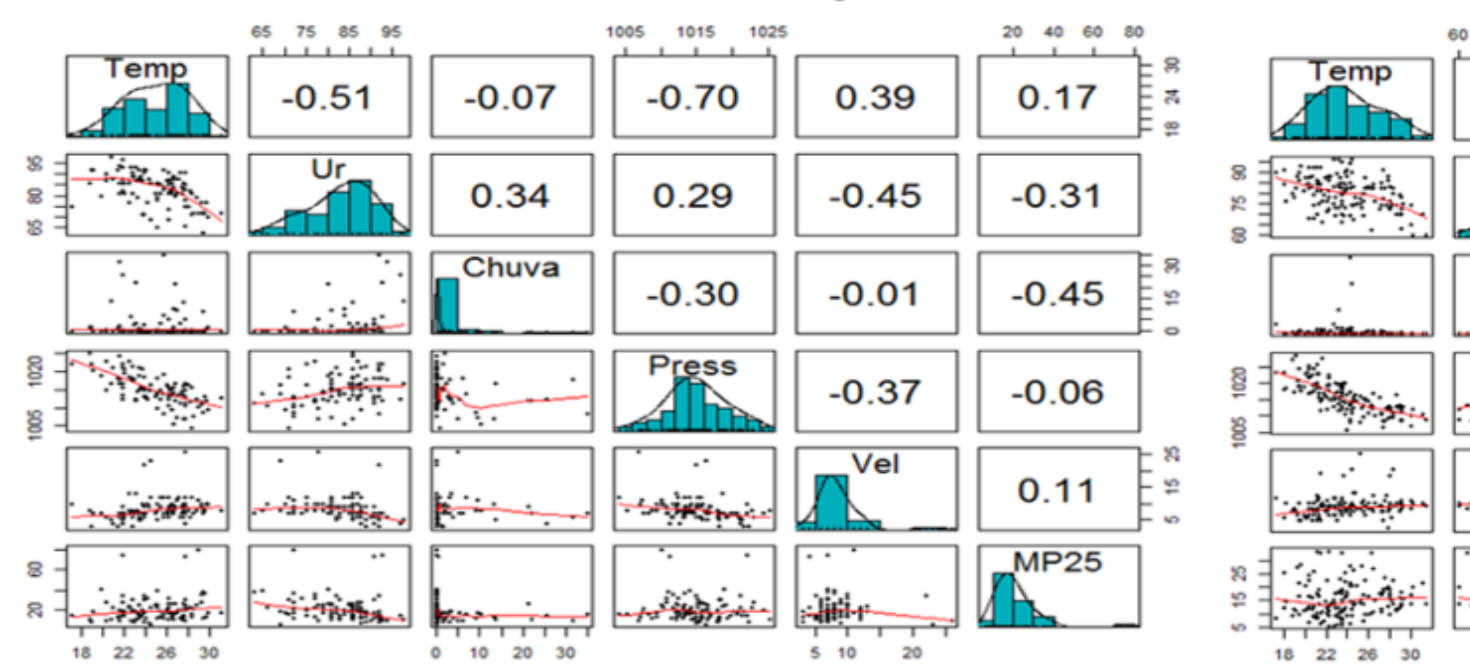

EMQA Rio de Grande - Operação

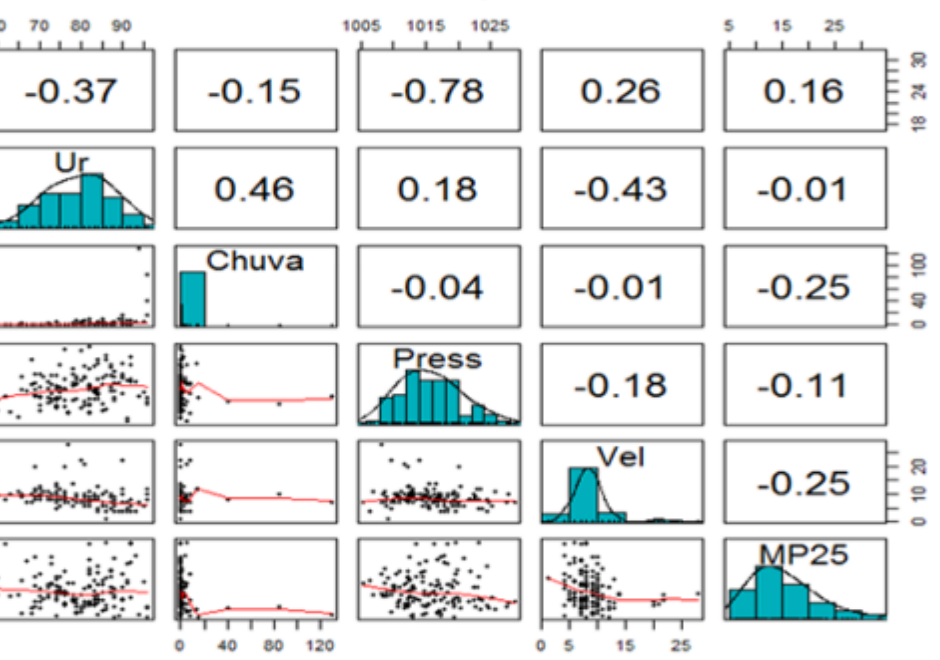

Figura 2 - Correlação entre a concentração de $\mathrm{MP}_{2,5}(\mathrm{MP} 25)$ e as variáveis meteorológicas nas EMQQA. Fonte: 0s Autores. 


\section{CONCLUSÃO}

A análise dos dados de monitoramento da concentração de $\mathrm{MP}_{2,5}$ em duas EMQA próximas ao corredor viário Transolímpica, durante o período de 14 de outubro de 2014 a 28 de outubro de 2018, mostrou que em três dos 106 dias amostrados foi violado o valor limite para $\mathrm{MP}_{2,5}$ estabelecido pela Resolução CONAMA N ${ }^{\circ}$ 491/2018, de $60 \mu \mathrm{g} \mathrm{m}^{-3}$. Isso aconteceu apenas na EMQQA Rio Grande, durante a fase de instalação. Entretanto, o padrão estabelecido pela OMS foi extrapolado em 81 dias de amostragem de um total de 246 dias, considerando as fases de instalação e operação da Transolímpica nas EMQ̧A Rio Grande e Bosque da Boiuna.

A concentração de $\mathrm{MP}_{2,5}$ na fase de instalação apresentou diferença estatisticamente significativa quando comparada com a fase de operação da Transolímpica. Para ambas as EMQA avaliadas a concentração de $\mathrm{MP}_{2,5}$ foi maior na fase obras. A principal hipótese para explicar esse resultado é que apesar das obras gerarem mais $\mathrm{MP}_{10}$ o fluxo de veículos, em especial os movidos a diesel, que emite mais $\mathrm{MP}_{2,5}$, é muito maior na fase de obras do que na fase de operação da via.

A correlação entre a concentração de $\mathrm{MP}_{2,5}$ e as variáveis meteorológicas se mostrou fraca de forma geral, sendo a precipitação pluviométrica a variável com maior valor de coeficiente de correlação de Spearman e, consequentemente, maior associação com o $\mathrm{MP}_{2,5}$. Precipitação pluviométrica, umidade relativa do ar e pressão atmosférica apresentaram correlação negativa com a concentração de $\mathrm{MP}_{2,5}$. Enquanto a temperatura exibiu correlação positiva com a concentração de $\mathrm{MP}_{2,5}$, a velocidade do vento não apresentou um padrão comum de correlação com $\mathrm{MP}_{2,5}$.

\section{REFERÊNCIAS BIBLIOGRÁFICAS}

BRASIL. Resolução CONAMA n 491 de 19 de novembro de 2018. Dispõe sobre padrões de qualidade do ar. Brasília (DF), 2018. Disponível em: < http://www2.mma.gov.br/port/conama/legiabre.cfm?codlegi=740>. Acessado em março/2019. 2018.

CHOWDHURI, A.; GUPTA, C. K. Assessment of particulate matter (PM) concentrations at a typical construction site in Bangalore, India. International Research Journal of Environment Sciences, v. 6, n. 2, 2017, p. 14-18.

DIAS, J. W. C. Manual de 0peração do Amostrador de Grande Volume (AGV) para partículas de até 2,5um (MP 2,5$)$. Energética Qualidade do Ar, 2017, 117 p. Disponível em: http://www.energetica.ind.br/wp/envl/wpcontent/uploads/2016/01/envl_manual-mp25_rev_06.pdf. Acesso em: 26 jan. 2018.

GUARIEIRO, L. L. N.; VASCONCELLOS, P. C.; SOLCI, M. C. Air Pollutants from the Burning of Fossil Fuels and Biofuels: A Brief Review. Revista Virtual de Química, v. 3, n. 5, 2011, p. 434-445. 
GUERRA, F.P.; MIRANDA, R.M. (2011) Influência da meteorologia na concentração do poluente atmosférico PM2,5 na RMRJ e na RMSP. In: II Congresso Brasileiro de Gestão Ambiental, Londrina. Anais. Disponível em: https://www.ibeas.org.br/congresso/Trabalhos2011/IV-007.pdf. Acesso em: 26 jan. 2018.

G0DOY, M. L. D. P., ALMEIDA, A. C., TONIETTO, G. B., \& G0DOY, J. M. (2018). Fine and coarse aerosol at Rio de Janeiro prior to the Olympic Games: chemical composition and source apportionment. Journal of the Brazilian Chemical Society, v. 29, n. 3, p. 499-508.

INEA. Instituto Estadual do Ambiente. Diretoria de Licenciamento Ambiental. Parecer Técnico de Licença de Instalação $\mathrm{N}^{\mathbf{0}} 18 / 13,2013,30$ p.

JERRET, M. Atmospheric science: the death toll from air-pollution sources. Nature, n. 525, 2015, p. 330-331.

JUDA-REZLER, K.; REIZER, M.; OUDINET, J. P. Determination and analysis of PM10 source apportionment during episodes of air pollution in Central Eastern European urban areas: The case of wintertime 2006. Atmospheric Environment, v. 45, n. 36, 2011, p. 6557-6566.

KINSEY, J. S.; LINNA, K. J.; SQUIER, W. C.; MULESKI, G. E.; COWHERD JR, C. Characterization of the fugitive particulate emissions from construction mud/dirt carryout. Journal of the Air and Waste Management Association, v. 54, n. 11, 2004, p. 1394-1404.

LELIEVELD, J.; EVANS, J.S.; FNAIS, M.; GIANNADAKI, D.; POZZER, A. The contribution of outdoor air pollution sources to premature mortality on a global scale. Nature, n. 525, 2015, 367-371.

LIU, Z.; HU, B.; ZHANG, J.; XIN, J.; WU, F.; GAO, W.; WANG, M.; WANG, Y. Characterization of fine particles during the 2014 Asia-Pacific economic cooperation summit: Number concentration, size distribution and sources. Tellus, Series B: Chemical and Physical Meteorology, v. 69, n. 1, 2017.

MARTINS, E. M.; FORTES, J. D. N.; RIBEIR0, G. P.; PEREIRA, M. F. M. Utilização de Sistema de Informação Geográfica como ferramenta para gestão do monitoramento da qualidade do ar na Região Metropolitana do Rio de Janeiro. Engenharia Sanitaria e Ambiental, v. 19, n. spe, 2014, p. 43-50.

MING, L.; JIN, L.; LI, JUN.; FU, P.; YANG, W.; LIU, D.; ZHANG, G.; WANG, Z.; LI, X. PM2.5in the Yangtze River Delta, China: Chemical compositions, seasonal variations, and regional pollution events. Environmental Pollution, v. 223, 2017, p. 200-212.

MONTE, E Z;; ALBUQUERQUE, T. T. A.; REISEN, V. A.; Impactos das Variáveis Meteorológicas na Qualidade do Ar da Região da Grande Vitória, Espírito Santo, Brasil Impacts of Meteorological Variables on Air Quality in The Region of. Revista Brasileira de Meteorologia, 2016, p. 546-554.

SÁNCHEZ, L.E. Avaliação de Impacto Ambiental: conceitos e métodos, 2 ed. São Paulo: Oficina de Textos, 2013,583 p.

VENTURA, L. M. B. Avaliação quimiométrica do comportamento do material particulado fino na atmosfera no estado do Rio de Janeiro. Tese (doutorado) - Pontifícia Universidade Católica do Rio de Janeiro, Departamento de Química, 2015 , p.149 - 223 .

VENTURA, L. M. B.; RAMOS, M. B.; GIODA, A.; FRANÇA, B. B.; DE OLIVEIRA GODOY, J. M. Air quality monitoring assessment during the 2016 Olympic Games in Rio de Janeiro, Brazil. Environmental monitoring and assessment, v. 191, p. 369-205, 2019. 